\title{
TECHNOLOGY FEATURE LIFE IN THE DANGER ZONE
}

\section{Instruments for studying microbes under biological containment cannot be readily removed from labs for servicing. A US facility is finding ways around that problem.}

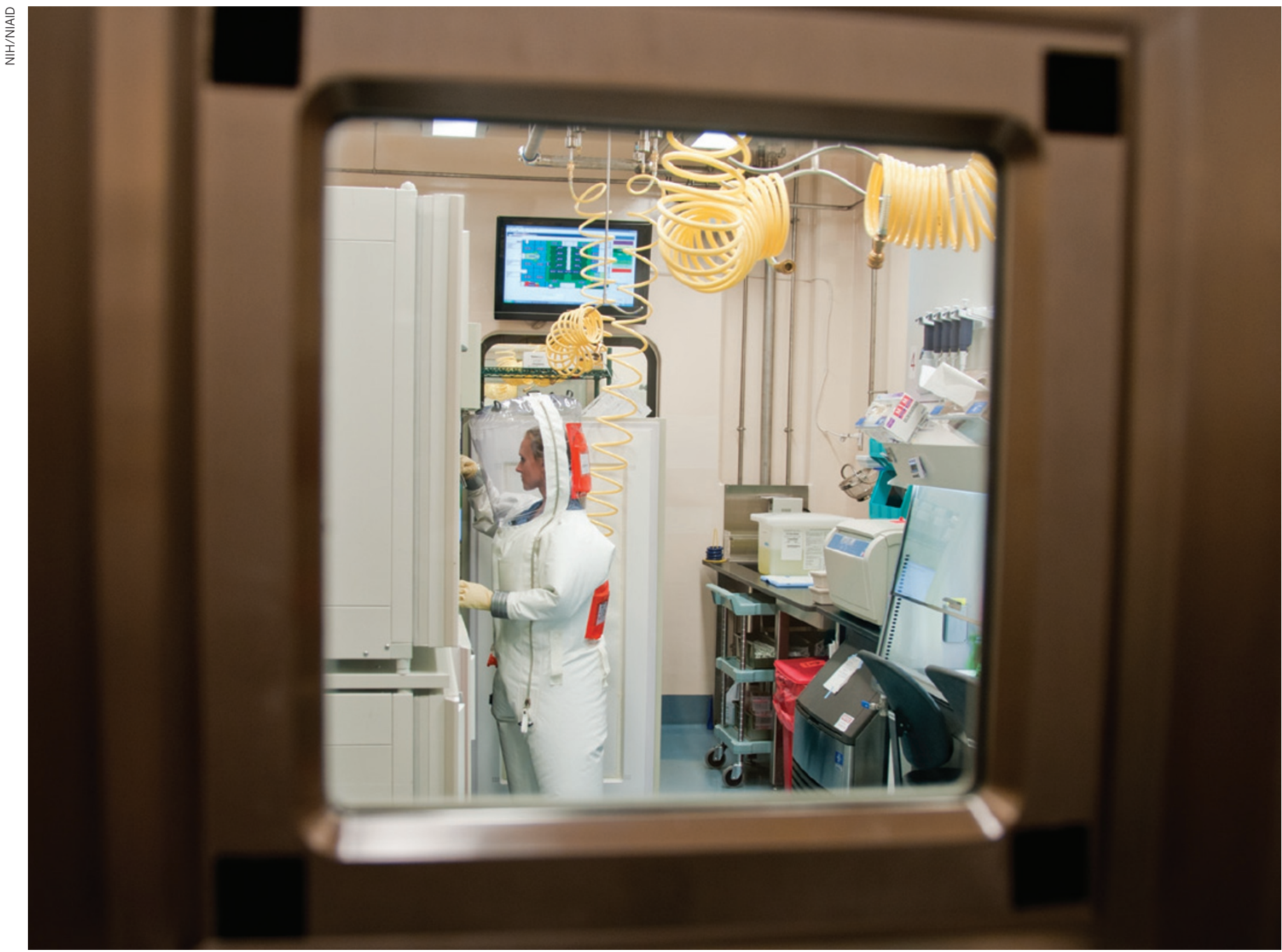

A laboratory in Frederick, Maryland, will soon bring a high degree of automation and imaging capability to research under biosafety-level-4 conditions.

\section{BY VIVIEN MARX}

ome bacteria, viruses and toxins are deadly - natural threats to humans $\checkmark$ and the environment as well as potential bioweapons. To counter such hazards, laboratories that study these pathogens and substances must do so under high security. One such lab is opening this spring in Frederick, Maryland. It will be designated as biosafety-level-4 (BSL-4), the highest level of biological containment.

The lab is part of the Integrated Research Facility (IRF), a complex operated by the US
National Institute of Allergy and Infectious Diseases (NIAID) that has been opening in stages since 2008. Although some of the equipment in the 1,020-square-metre lab is standard for a BSL-4 environment, the degree of automation and integration is unprecedented, says Peter Jahrling, director of the IRF.

For example, a complete imaging suite and clinical area lets researchers study and treat infected animals without having to remove them from containment. "We've basically built an intensive care unit for animals," says Jahrling. Likewise, the instruments used to screen and study blood and tissue samples have been customized to minimize the need for handling by people. In some cases, almost every step has been automated, with robots doing assays to check virus concentrations or assess immune responses.

Until the lab receives its final BSL-4 approval, which is expected early this year, its staff is working on slightly less dangerous microbes, such as the viruses that cause cowpox and Middle East respiratory syndrome (MERS). But the researchers are already following BSL-4 procedures - wearing pressurized whole-body suits with air piped in through hoses, and taking disinfecting $>$ 

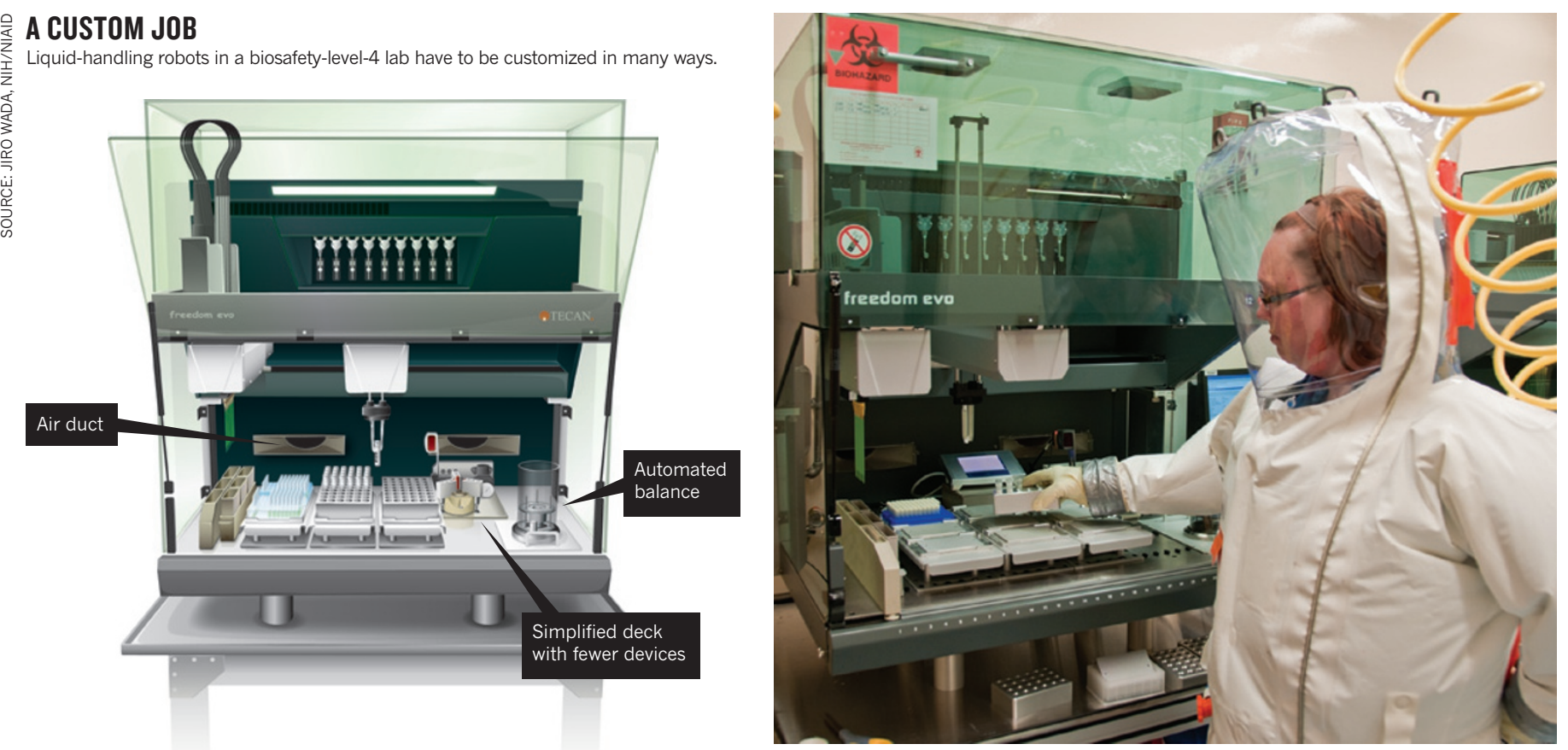

A liquid-handling robot designed for use by scientists in bulky protective gear.

chemical showers in the suits when exiting the lab. "It allows our staff here to gain practice in working with a human pathogen in a BSL-4 environment," says Lisa Hensley, a microbiologist and the IRF's associate research director.

Once the lab has final approval, Hensley and her team will move on to study the Ebola, Hendra and Nipah viruses. "We'll be able to see what's really happening in animals," she says, and to assess potential treatments. The lab's unique ability to image live specimens in the BSL-4 environment will help researchers to cut down on the number of animals killed, and will avoid the need for extensive and numerous post-mortem examinations, which increase the risk to staff.

But installing robots and complex imaging equipment in the lab has called for careful engineering.

\section{Clean IMaging}

In 2012, scientists at another NIAID BSL-4 lab, in Hamilton, Montana, showed that the infection that causes MERS in humans could be modelled in macaque monkeys (V. J. Munster et al. N. Engl. J. Med. 368, 1560-1562; 2013). The scientists there could do blood work on these animals and take X-rays, says Hensley, but more detailed imaging data are crucial for identifying lung regions affected by the disease, pinpointing possible locations where inflammation begins and assessing possible treatments. "You can look to see if your countermeasure is working," says Hensley.

That is why the new IRF lab has a hospitalquality imaging suite that includes an X-ray imager, a 3-tesla magnetic resonance imaging machine and scanners that combine computed tomography with positron emission tomography or single-photon emission computed

tomography (see 'Hot and cold').

But each piece of equipment has many moving parts and electronic components that need regular servicing, says Jahrling. "There's no way you can put that in a BSL-4 lab and expect it to function for very long," he says. And in a lab with such high biosafety requirements, "the service people aren't coming in", says Hensley. The equipment lives in containment and can leave only if thoroughly decontaminated, yet no instrument can handle a chemical shower. The alternative - treatment with vaporous formaldehyde or hydrogen peroxide - can also cause damage. And even if the instrument were to survive, the process would be time-consuming.

So before the IRF scientists installed imaging equipment in the BSL-4 lab, they collabo-

\section{"The more}

things you add to

a robot, the more

things can go

wrong." rated with engineers at Philips Healthcare, which has its US headquarters in Andover, Massachusetts, to completely re-engineer the instruments. "That was a huge research and development effort," says Jahrling.

The main sections of the imaging machines are installed on one side of a wall - the 'cold' or non-contained side. On the 'hot' side is a patient table onto which an anaesthetized animal is placed for imaging. The table is mounted on tracks and can be moved through a secure transparent tube that extends into the cold side of the lab but is sealed to prevent the escape of pathogens. Researchers then slide the table through the tube to the imaging apparatus on the cold side. This design allows service staff to maintain the instrument outside the contained lab.
Four liquid-handling robots have been installed at the IRF, two of which are in the BSL-4 lab. The instruments are used to extract DNA and RNA from tissues and to perform assays that involve titration and staining. The robots cap and uncap tubes, weigh them and dispense various reagents.

In plaque assays, used for calculating the concentration of virus in a sample, virus is added to a multiple-well plate that contains cells and culture medium. After a certain amount of time, visible holes or 'plaques' form where the virus has infected and killed cells. The assay requires multiple steps that include serial dilution, weighing samples and adding buffers.

In neutralization assays, used for assessing immune responses, scientists can see how well antibodies interfere with an infectious agent. When robots tend to these assays, there is less variability than when a number of different staff members perform the task, says Hensley. Less handling of infectious and toxic agents is also safer for scientists, she says.

\section{SIMPLIFIED ROBOTS}

Automation brings its own problems. For example, Jahrling says, "there's a concern that the repetitive manipulations that the robot performs might generate an aerosol" - tiny, airborne droplets that can spread infectious agents. To reduce the risk, some instruments were enclosed and tested to ensure that performance was not impaired.

Kenny Ung, an engineer at Tecan, a company based in Männedorf, Switzerland, and his colleagues worked with the IRF team to customize Tecan's Freedom EVO liquid handlers for use in the lab. The robots are enclosed by safety shields. 
Tecan customizes instruments for complex workflows in which robotic arms move between carousels, bar-code scanners, incubators, shakers, sealers and other devices. But the machines needed to be simplified and customized for use in this lab. Given the space constraints and difficulty servicing instruments, Ung says, "you don't want one of those types of systems inside BSL-4". But the IRF team wanted some degree of automation in their assays so that many samples can be prepared at once. "A robot doesn't get bored, doesn't get tired," says Ung.

In the IRF's BSL-4 lab, robots are programmed for sample preparation with fewer additional devices and with as little human intervention as possible (see 'A custom job'). A robotic arm will pick up tubes containing tissue or blood samples, move them to an automated balance, uncap and cap them and add buffer as needed. Another robot dispenses liquids for serial dilution of samples, which are then transferred to 96-well plates to be incubated and analysed.

Tecan's engineers customized the liquid handler to deal with this succession of tasks, but kept the robot simple. They added an automated balance and a bar-code reader to scan and keep track of the sample tubes, but decided not to include a sample-mixing sonicator instead, the IRF scientists use an external mixer. "The more things you add to a robot, the more things can go wrong," Ung says which is true especially in a BSL-4 environment, where repair needs must be kept to a minimum.

Ung knew that scientists would be operating the instrument while wearing protective suits, so he decided to simulate their

situation. "I wanted to feel what it's like to be the user inside the lab," he says. The IRF team sent him a safety suit to wear while he worked on the machine.

One of Ung's tasks was to ensure that the equipment had no sharp edges that could

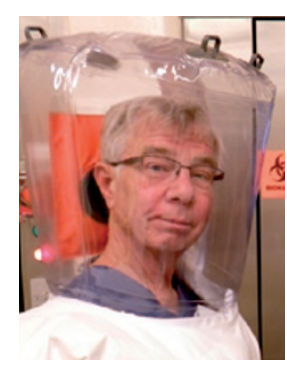

"Youjust get used to disconnecting your air and walling ten feet down to the next air line and reconnecting."

Peter Jahrling as to disrupt liquid-dispensing or weighing. Increased air flow can prevent a balance from stabilizing, which would lead to flawed readings.

Before installing the machine, the IRF team visited Tecan's labs to verify that it worked as specified. The instrument was then moved to the BSL-4 lab, where it was reassembled and tested. Tecan worked out a maintenance plan for the instrument. "I'm not able to go in there, so they basically need to have skills on their side to remedy the problem," says Ung. Cindy
Allan, a biomedical engineer at the IRF, has learned how to take the robot apart and put it back together. She also learned the tricky task of changing the pipette tips, diluters and syringes. Biothreat analysis calls for genetic and protein-based tests, says Amy Altman, who directs biodefence at Luminex, an instrument manufacturer in Austin, Texas. Antibody-based probes are used to detect proteins such as ricin or Clostridium botulinum toxin, for example. And some viruses can have very low concentrations in the blood, so detecting them requires sensitive genomic tests.

\section{FAST FLOW}

In the IRF's BSL-4 lab, researchers will be using Luminex's FlexMAP 3D, which is based on flow cytometry, a technique in which lasers are used to count cells. In this instrument, specific types of genetic material or protein are attached to beads coloured with multiple fluorescent dyes at different ratios. The beads move in single file past a laser that emits red light to excite the dyes. The emitted wavelength of light identifies the type of bead. A green laser then excites a 'reporter' dye in the bead that determines which protein or nucleic acid it is attached to. "As each bead gets interrogated by the laser, you can think of each bead as its own little test tube," says Altman.

To screen a sample for ten strains of Ebola virus, for example, researchers use an array of beads that each carry snippets of DNA specific to one of the strains. A blood sample from a patient who succumbed to Ebola can therefore be tested to determine which strain caused that person's death.

The platform works quickly and can distinguish between up to 500 genes or proteins

\section{HOT AND COLD}

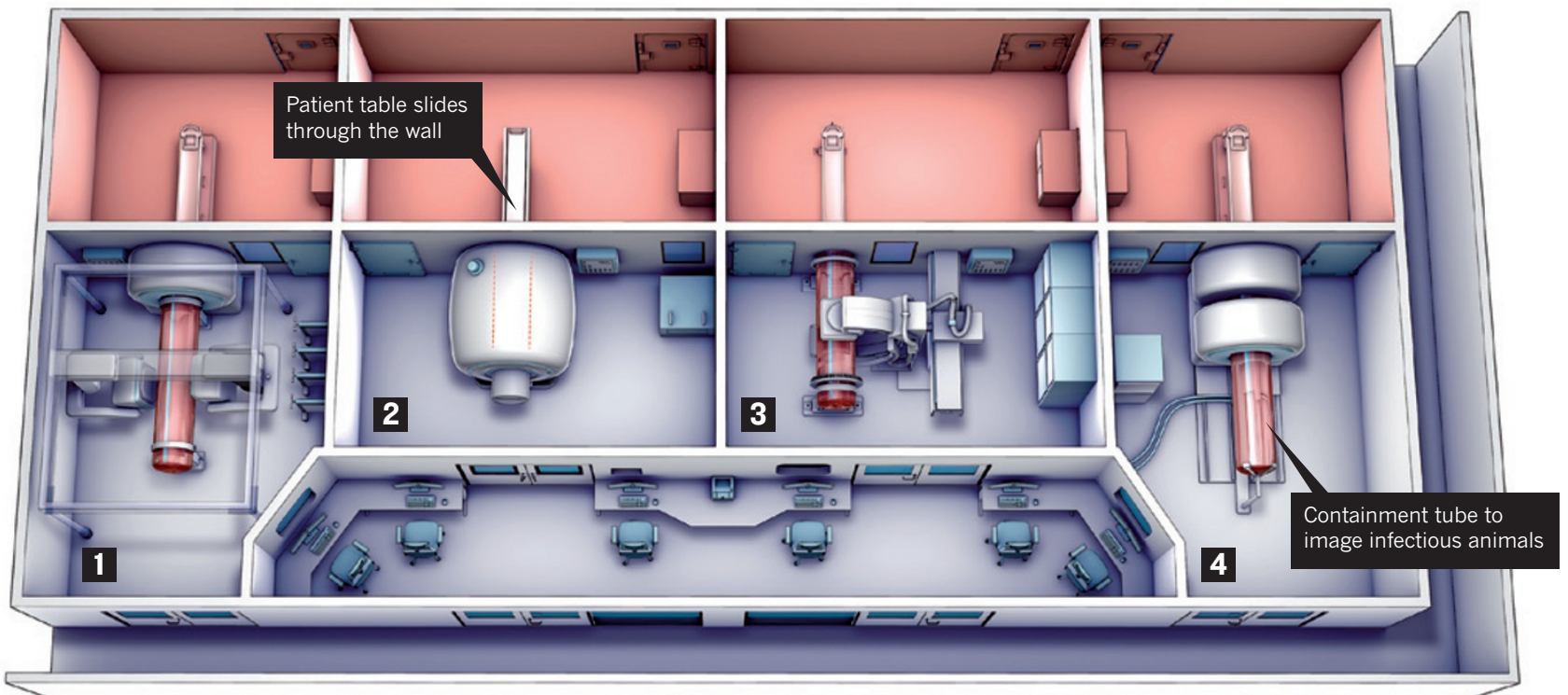

Sing

Single-photon emission computed tomography and computed tomography (SPECT-CT)

Magnetic resonance imaging (MRI)

3 X-ray imaging

4 Positron emission tomography and computed tomography (PET-CT) 
simultaneously. Such 'multiplexing' means that scientists can check for many signatures of a possible infectious agent at once, Altman says; a 'true positive' for a pathogen would be when all signatures of the disease agent are present. Multiplexing platforms are used in biothreat monitoring, which sometimes entails "highconsequence, high-regret decisions", such as whether or not to shut down an airport, says Altman.

To ensure that the hardware could operate in the IRF's BSL-4 lab, Luminex scientists tested whether the instrument could be safely decontaminated with vaporous hydrogen peroxide and paraformaldehyde. Neither substance is usually kind to instruments, but in the case of the Luminex machine, "it made it through fine", Altman says.

\section{BACTERIAL HIGH ALERT}

Scientists in the BSL-4 lab will also be using the BacT/ALERT 3D 60, an automated system made by bioMérieux, based in Marcy-l'Étoile, France, that tests samples of body fluid for fungi, mycobacteria or bacteria - such as Yersinia pestis, which causes bubonic plague, or the anthrax culprit Bacillus anthracis.

To accomplish this task, the instrument has a built-in incubator that rocks sample bottles, swirling their contents to enhance microbial growth. At the bottom of each bottle is a dab of dried silicone that contains a dye and acts as a $\mathrm{pH}$ sensor. When the $\mathrm{pH}$ in a bottle changes because of microbial respiration, the sensor's colour changes from blue-green to yellow. A detection system illuminates the bottles periodically and alerts lab staff to any change in sensor colour, says Doug Matthews, product manager at bioMérieux.

"Every ten minutes, it takes a reading and will alert you if it detects growth," Matthews says. Speed is important in hospitals, where this instrument is often deployed, as well as in clinical-testing labs and the pharmaceutical and food industries. But in a BSL-4 lab, rapid detection is even more crucial, he says.

After detection through this automated incubator, scientists can then confirm their findings with other steps. A sample will be grown in an agar dish to identify specific pathogens, for example, or to test how well an antimicrobial agent might work. Researchers might also extract genetic material for sequencing.

The BacT/ALERT 3D 60 was not made specifically for BSL-4 labs, but a number of its design features help it to work in that environment, says Matthews. Despite its compact size, it can handle 60 samples. Its touch screen can be operated by heavily gloved hands. The instrument is designed to be hardy - company records of installed instruments show that the machines do not need major service or repair for an average of 1,000 days, he says. The sample bottles are made from nylon sandwiched

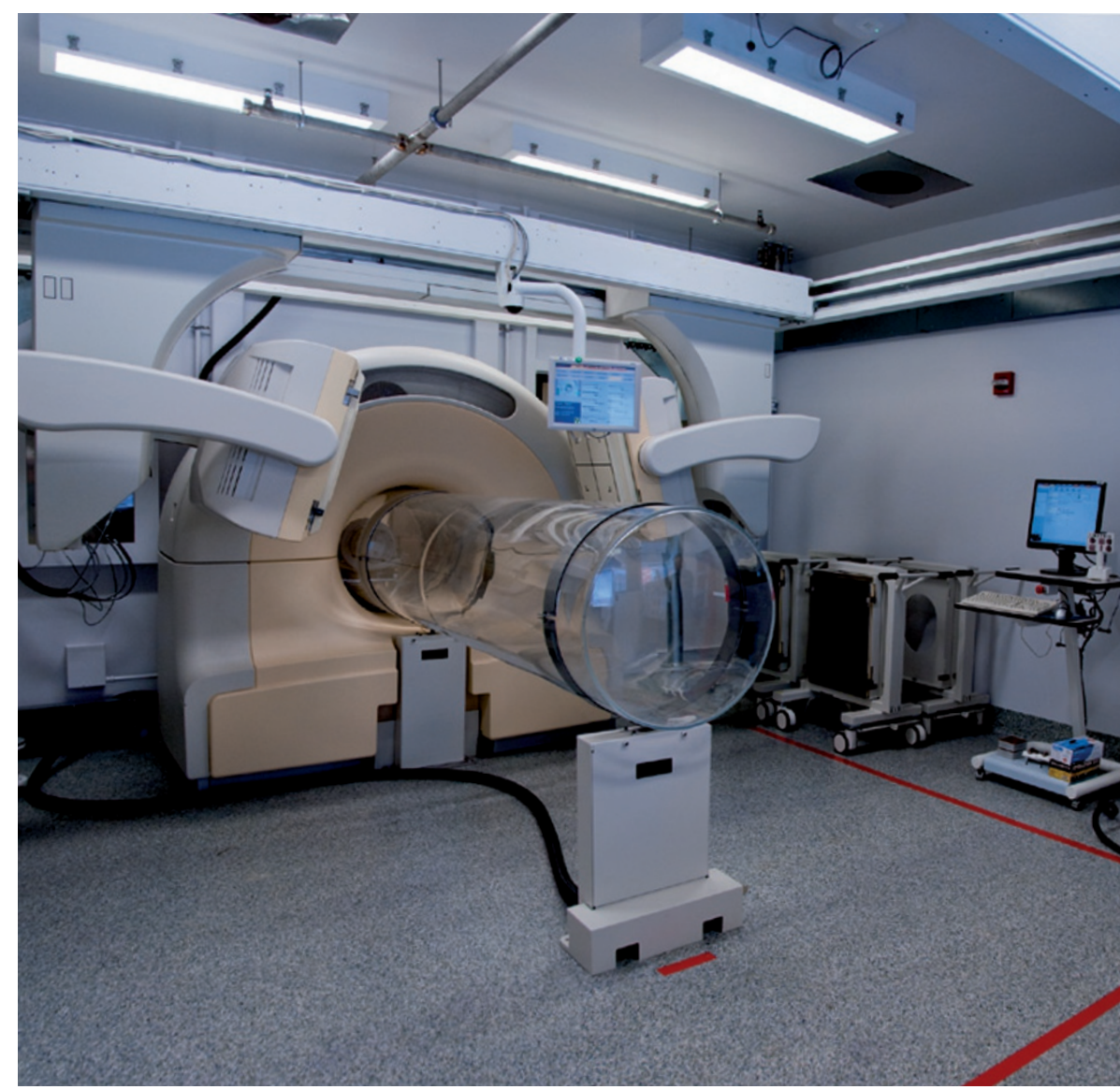

In a custom computed-tomography scanner, animals can be imaged without contaminating equipment.

between two layers of polycarbonate to resist shattering and spreading infectious cultures. "Keeping glass out of a BSL lab is obviously a key safety feature," Matthews says.

Later this year, bioMérieux intends to launch a new model of the instrument, called the Virtuo, in Europe. The Virtuo handles more bottles than the BacT/ALERT 3D 60 and automatically places them in racks after scanning their bar codes. The instrument is now in clinical trials, says Matthews, and the company is slated to file for approval with the US Food and Drug Administration in 2015.

\section{CELLULAR CONNECTION}

The IRF's new BSL-4 lab is subdivided into rooms for different tasks, such as sample preparation, cell culture, animal care or imaging, and tests for clinical pathology, molecular biology and virology. But moving between those rooms is not simple, because scientists in protective gear are tethered by an air hose that stretches only so far. To venture farther, they have to detach from their air supply. "If you disconnect your air you could last for ten minutes," says Jahrling. "You just get used to disconnecting your air and walking ten feet down to the next air line and reconnecting."

Given the multitude of tasks researchers do in the IRF lab, they need to confer often. Years of working in 'space suits' have made Hensley and Jahrling fluent in non-verbal communication
- by a wave of a gloved hand, for example. For the new lab, they wanted to go one better - in part because they noted a cultural shift among younger scientists, who seemed to want to communicate more when in the BSL-4 lab.

After testing various communication systems, they chose mobile phones and Bluetooth headsets. The phones are external to the suits; the headsets are internal. "You pick up the cell phone on the hot side of the suit," Hensley says.

Communication and collaboration have been a goal for the lab since 2005, when construction of the IRF began as part of a plan to bolster US biodefence research. To enable collaboration, the IRF is located next to the US Army Medical Research Institute of Infectious Diseases (USAMRIID) and labs run by the US Department of Homeland Security. Both Jahrling and Hensley previously worked at the USAMRIID.

Jahrling says that those neighbouring labs are the subject of much rumour and speculation, but that no secret research is done at the IRF. On the contrary, he has extended an open invitation to scientists from other biosafety research facilities to visit, so that they can see for themselves what it is like to battle the most noxious weapons from nature's arsenal in an up-and-running, next-generation BSL-4 lab..

Vivien Marx is technology editor for Nature and Nature Methods. 